epine management; all returned to baseline psychiatric function within the same day.

Conclusions.-The near-simultaneous and unexpected onset of 7 cases of acute anxiety, coincident with new arrival at moderate to high altitude, represents a highly unusual incidence, prevalence, and temporal distribution suggestive of hypobaric hypoxemia as the proximal cause. Based upon the authors' extensive combined clinical experience and review of emerging peer-reviewed literature, High Altitude Anxiety is proposed as a diagnostic entity for consideration in the Diagnostic and Statistical Manual of Mental Disorders (DSM-5). Further research is indicated.

\author{
Jeffrey H. Gertsch, MD \\ Erik Virre, MD \\ La Jolla, CA, USA
}

LT Michael K. Sracic, MD, MC, USN HMC Allen Pate, IDC, USN Camp Pendleton, CA, USA

LT Darren Thomas, MD, MC, USN

Bridgeport, CA, USA

\section{Exercise-associated Hyponatremia is Common Among Dehydrated 161 Kilometer Ultramarathoners}

Objective.-Previous work has demonstrated that exerciseassociated hyponatremia (EAH) rarely occurs in association with dehydration and that there is a significant negative relationship between post-race serum sodium concentration $\left(\mathrm{Na}^{+}\right)$ and weight change. In other words, EAH has been found to occur more often when there is weight gain rather than weight loss during the event. This has been in contrast to our observations from $161 \mathrm{~km}$ ultramarathon races in northern California. The present work consolidates our findings from studies at three $161 \mathrm{~km}$ ultramarathons in northern California.

Methods.-Data were compiled from studies performed at the 2008 Rio Del Lago, and the 2009 and 2010 Western States Endurance Run, all $161 \mathrm{~km}$ trail footraces in northern California. Consenting runners underwent prerace and postrace measurement of body weight on the same calibrated scale. Sodium concentration was measured on-site immediately postrace using an iSTAT (Abbott, NJ) analyzer.

Results.-An EAH incidence of $30 \%$ to $51 \%$ was observed at the 3 events. Overall, EAH was present in 83 (33.3\%) of 249 observations. In each case of EAH, the runner appeared neurologically intact. Of the 223 observations in which prerace and postrace body weights were also obtained, 41 (18.4\%) were overhydrated (weight change $\geq 0 \%$ ), 99 (44.4\%) were euhydrated (weight change $-3 \%$ to $0 \%$ ), and 83 (37.2\%) were dehydrated (weight change $<-3 \%$ ). Biochemical EAH $\left(\mathrm{Na}^{+}\right.$ 129 to $<135 \mathrm{mmol} / \mathrm{L}$ ) was present among $65(29.1 \%)$ and "clinically significant" EAH $\left(\mathrm{Na}^{+}<129 \mathrm{mmol} / \mathrm{L}\right)$ was present among $13(5.8 \%)$. Considering the 78 with EAH, 35 (44.9\%) were dehydrated. Of the 13 with "clinically significant" EAH, $4(30.8 \%)$ were dehydrated. Spearman correlation between postrace $\mathrm{Na}^{+}$and percentage change in body weight was significant $(r=0.14, P=.037)$.
Conclusions.-The $\geq 30 \%$ incidence of EAH among participants in $161 \mathrm{~km}$ ultramarathons in northern California is among the highest rates reported from any event. Unlike the findings from previous work, EAH was commonly observed in association with dehydration. As such, in this group of ultramarathoners, significant weight loss cannot be used to exclude the presence of EAH.

Martin D. Hoffman, MD Sacramento, CA, USA

Tamara Hew-Butler, DPM, PhD Rochester, MI, USA

Kristin J. Stuempfle, PhD Gettysburg, PA, USA

Ian R. Rogers, MBBS Perth, Australia

\section{Wilderness Medicine Within Global Health: A Strategy for Less Risk and More Reward}

A Case Study of Integrating the 2 Disciplines at Weill Cornell Medical College

Objective.-Over the last decade, there has been a growing interest in global health among medical students. According to the Association of American Medical Colleges, 45 US medical schools offer a Global Health component, with $29.9 \%$ of graduating students stating they have had "global health experience." With an emphasis on self-reliance and the delivery of medical care in austere and unpredictable environments, we advocate that wilderness medicine (WM) skill sets are an integral component of global heath, mitigating risk to students and empowering them to be safe as they participate in this rapidly evolving field. Our objective was to examine ways in which WM topics can be incorporated into global health coursework, and the benefits that come from this integration.

Methods.-In 2008, Weill Cornell Medical College (WCMC) students and faculty designed a 4-year Global Health Curriculum (GHC). The core competencies of GHC are intended to complement and expand upon required pre-existing global health lectures. In designing our "Clinical Skills for Resource-Poor Environments" elective offered to 4th-year medical students, the aim was to prepare students for going abroad by instilling skills that would reinforce self-sufficiency and well being. Approximately $15 \%$ of the WM curriculum was incorporated into the 2-week, 40-hour Clinical Skills course through lectures, hands-on labs, workshops, and skills-based assessments taught by over 25 faculty members throughout New York City.

Results.-The course has been offered twice since February 2010. Evaluations of individual lectures and labs provided helpful qualitative and quantitative feedback. All participants would recommend the course to others. Many claimed that the course offered hands-on skills training that was not otherwise addressed in their medical education.

Conclusions.-The Weill Cornell GHC elective, "Clinical Skills for Resource-Poor Environments," demonstrates an in- 